J. F. McClendon

Nagoya Math. J.

Vol. 54 (1974), 149-164

\title{
REDUCING TOWERS OF PRINCIPAL FIBRATIONS
}

\author{
J. F. MCCLENDON
}

Consider a tower of principal fibrations

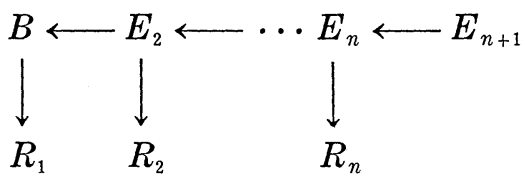

That is, $E_{i+1}$ is the pullback of $E_{i} \rightarrow R_{i}$ and the path fibration $P R_{i} \rightarrow R_{i}$. The question arises as to whether or not the tower can be shortened, that is, whether or not $E_{n+1} \rightarrow B$ is fiber homotopically equivalent to a nice fibration $E \rightarrow B$. If "nice" is taken to mean "principal" then sufficient conditions are known. They involve connectivity assumptions on the $E_{i}$. In this paper "nice" is taken to mean " $D$-relatively principal" for some space $D$. Relative principal fibrations are more general than principal fibrations. Their lifting properties are studied in [7]. They enjoy some but not all of the nice properties of principal fibrations. The assumptions on the tower above which imply that $E_{n+1} \rightarrow B$ is nice are weaker than the assumptions showing it to be principal-as expected, since the conclusion is weaker.

One application of the sufficient conditions is a kind of represetation theorem for certain fibrations. Suppose $F \rightarrow E \rightarrow B$ is a fibration, $F, E$, $B$, having the homotopy type of $C W$ complexes, and $\Pi_{i}(F)=0$ except possibly when $s \leq i<2 s-1$. Then it is shown that $E \rightarrow B$ is a relatively principal fibration. No connectively assumptions are made on $B$. It follows that if $E \rightarrow B$ is any fibration with an $n$-connected fiber then the $2 n^{\prime}$ th stage of its Moore-Postnikov factorization is a relatively principal fibration.

In the first section a twisted suspension operation is studied. In the Received April 26, 1972.

0) Primary AMS classification numbers: 55F15, 55G35, 55G40, 55G45. Secondary AMS classification number 55E35.

1) This research was partially supported by NSF grant GP-14364. 
second section this operation is used to give sufficient conditions for reducing a two stage tower. In the last section sufficient conditions are given for reducing an arbitrary tower and the above mentioned representation theorem is proved.

\section{A Suspension Operation for Relatively Principal Fibrations.}

First, we recall a few definitions from [6]. Let Top $(u: C \rightarrow D)$ be the category of triples $(X, \check{x}, \hat{x})$ where $\check{x}: C \rightarrow X, \hat{x}: X \rightarrow D, \hat{x} \check{x}=u$ and all of this takes place in $\operatorname{Top}=\operatorname{Top}(\emptyset \rightarrow p t)=$ category of topological spaces and continuous functions. Write $\operatorname{Top}(D=D)$ for Top (id: $D \rightarrow D$ ). It has all of the basic properties of $\mathrm{Top}(\mathrm{pt}=\mathrm{pt})=$ the category of pointed spaces and maps. In particular if $Z \in \operatorname{Top}(D=D)$ then there is a canonical principal fibration (path-loop fibration) $\Omega_{D} Z \rightarrow P_{D} Z \rightarrow Z$. (The properties of Top $(C \rightarrow D)$ were established in my 1966 thesis [5] and outlined in the published abstract. A couple of years later similar notions were described by others.)

Now, let $X \in \operatorname{Top}(C \rightarrow D)$ and $f: X \rightarrow Z \in \operatorname{Top}(C \rightarrow D)$ where $C \rightarrow Z$ is $C \rightarrow D \rightarrow Z$. Then if $P=P_{f} \rightarrow X$ is the pullback of $P_{D} Z \rightarrow Z$ and $f$ then it is called a $D$-relative principal fibration. Suppose that $L \in \operatorname{Top}(D=D)$. We wish to define a secondary operation $\rho:[Z, L]_{D}^{D} \rightarrow$ $\left[P, \Omega_{D} L\right]_{D}^{C}$. The operation can be treated in a fairly direct manner. However, in the interest of clarity and unity (with an operation in [8]) we will start from an abstract level.

Consider the following data $(\Delta)$.

$$
\left\{\delta_{s}: H_{t} \longrightarrow G\right\} \quad S \stackrel{\beta}{\longrightarrow} T \stackrel{\alpha}{\longrightarrow}\left(U, u_{0}\right)
$$

Here $\left\{\delta_{s}: H_{t} \rightarrow G\right\}, t \in T, s \in \beta^{-1}(t)$, is a family of group homomorphisms. $S$ is a $G$-set, $\beta: S \rightarrow T, \alpha: T \rightarrow U$ are set maps, $u_{0} \in U, \alpha^{-1}\left(u_{0}\right)=\beta(S)$; each $\beta^{-1}(t)$ is a $G$-subset of $S$ and $G$ acts transitively on it; $\delta_{s}\left(H_{t}\right)=G_{s}$ $=\{g \in G \mid g s=s\}=$ the stability subgroup of $s . \quad \gamma_{s}: G \rightarrow S$ is defined by $\gamma(g)=g s$. This situation is exactly the one occurring at the bottom of exact homotopy sequences. A prototype example can be obtained as follows. Let $S$ be a $G$-set and $T=S / G, U=\left\{u_{0}\right\}, H_{t}=G_{s(t)}$ where $s(t)$ is a chosen element of $\beta^{-1}(t)$. For $s \in \beta^{-1}(t), \delta_{s}: H_{t} \rightarrow G$ is defined by $\delta_{s}(g)=\bar{g} g \bar{g}^{-1}$ where $s=\bar{g} s(t)$

Given the data $(\Delta)$, one can select $s \in S$ and form the sequence 


$$
\begin{aligned}
& H_{t} \stackrel{\delta_{s}}{\longrightarrow} G \stackrel{\gamma_{s}}{\longrightarrow} S \stackrel{\beta}{\longrightarrow} T \stackrel{\alpha}{\longrightarrow} U \\
& 1 \quad 1 \quad s \quad t=\beta s \quad u_{0}
\end{aligned}
$$

Then it is easily checked that this is an exact sequence of pointed sets.

A morphism $\Delta \rightarrow \Delta^{\prime}$ is
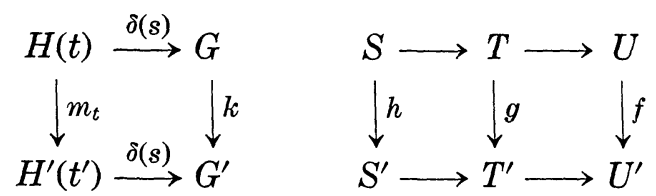

where the diagram is commutative, $g t=t^{\prime}, h s=s^{\prime}, m_{t}$ and $k$ are homomorphisms, and $f\left(u_{0}\right)=u_{0}^{\prime}$. For each $s \in S$ there is induced a morphism $\Delta(s) \rightarrow \Delta\left(s^{\prime}\right)$.

1.1 Definition. Let $\Delta \rightarrow \Delta^{\prime}$ be given. Let $t \in T, t^{\prime}=g t, \alpha t=u_{0}$. Suppose $s^{\prime} \in S^{\prime}$ with $\beta s^{\prime}=t^{\prime}$. Define

$$
\Gamma\left(s^{\prime} ; t\right)=\left\{g^{\prime} \in G^{\prime} \mid g^{\prime} s^{\prime} \in h \beta^{-1}(t)\right\} .
$$

1.2 THEOREM. (1) $\Gamma\left(s^{\prime} ; t\right)$ is a double coset of $\left(k G, G\left(s^{\prime}\right)\right)$, i.e., $g^{\prime} \in \Gamma\left(s^{\prime} ; t\right)$ implies $\Gamma\left(s^{\prime} ; t\right)=(k G) g^{\prime}\left(G\left(s^{\prime}\right)\right)$.

(2) $\Gamma\left(g^{\prime} s^{\prime} ; t\right) g^{\prime}=\Gamma\left(s^{\prime} ; t\right)$, all $g^{\prime} \in G^{\prime}$

(3) $k G$ normal in $G^{\prime}$ implies $\Gamma\left(g^{\prime} s^{\prime} ; t\right)=\Gamma\left(s^{\prime} ; t\right)$, all $g^{\prime} \in k(G)$

Proof. I'll prove (1) only. The interesting thing is that $s^{\prime}$ needn't be in the image of $h$. Pick $s_{0}$ with $\beta s_{0}=t$ so $\beta^{-1}(t)=G s_{0}$ and $h \beta^{-1} t=$ $h\left(G s_{0}\right)=(k G) h s_{0}$. Let $s_{0}^{\prime}=h s_{0}$. Suppose $g^{\prime}, g^{\prime \prime} \in \Gamma\left(s^{\prime} ; t\right)$ and $g^{\prime} s^{\prime}=k\left(g_{1}\right) s_{0}^{\prime}$, $g^{\prime \prime} s^{\prime}=k\left(g_{2}\right) s_{0}^{\prime}$. Thus $k g_{2}^{-1} g^{\prime \prime} s^{\prime}=k g_{1}^{-1} g^{\prime} s^{\prime}$, hence $\left(k g_{1}^{-1} g^{\prime}\right)^{-1} k g_{2}^{-1} g^{\prime \prime} \in G\left(s^{\prime}\right)$ implying $g^{\prime \prime} \in k g_{2} k g_{1}^{-1} g^{\prime} G\left(s^{\prime}\right) \subset k G g^{\prime} G(s)$. Conversely, $\bar{g} \in k G g^{\prime} G\left(s^{\prime}\right)$ implies $g^{\prime-1} k g^{-1} \bar{g} \in G\left(S^{\prime}\right) \quad$ (some $g$ ) implying $\quad \bar{g} s^{\prime}=k g g^{\prime} s^{\prime}=k g k g_{1} s_{0}^{\prime}$ and hence $\bar{g} \in \Gamma\left(s^{\prime} ; t\right)$.

As a first example we take up the operation of [8, Section 2]. Let $F \stackrel{f}{\longrightarrow} E \stackrel{g}{\longrightarrow} B$ be a fibration in Top (pt), $L \in \operatorname{Top}(D=D)$ and $h: B \rightarrow$ $L \in$ Top $($ pt $\rightarrow D$ ), putting $F \rightarrow E \rightarrow B$ into Top $($ pt $\rightarrow D$ ). Consider

$$
(F, F) \longrightarrow(E, F) \longrightarrow(B, \mathrm{pt}) \longrightarrow(L, L) .
$$

Theorem 3.4 of [7] and the obvious naturality give the following situation where [ ] means [ ] $]^{\mathrm{pt}}$. 


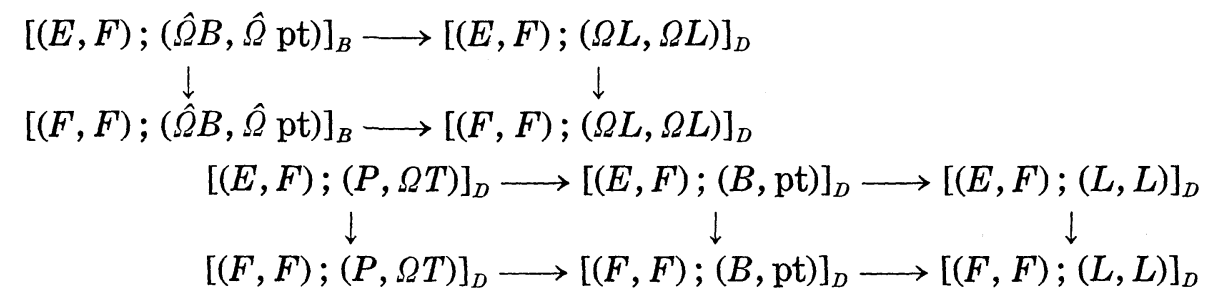

Here $P=P_{h}, T=\check{k}^{-1}\left(d_{0}\right)$ where $\check{k}: L \rightarrow D, \Omega T$ is the ordinary loop space, $\Omega L$ means $\Omega_{D} L$ and $\hat{\Omega}$ means $\hat{\Omega}_{D}$. This simplifies to

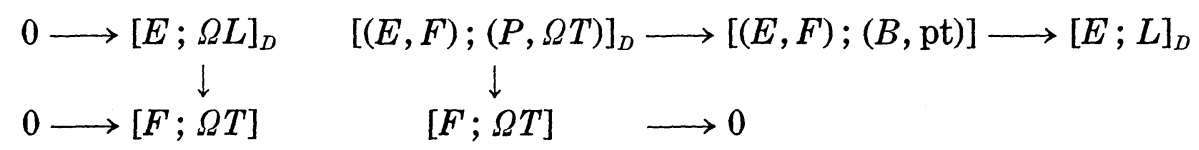

Take $s^{\prime}=*: F \rightarrow \Omega T$ and define $\sum:[(E, F) ;(B, \mathrm{pt})]_{D} \rightarrow[F ; \Omega T]$ by $\sum(g)$ $=\Gamma\left(s^{\prime} ; g\right)$. It follows from 1.2 that $\sum g$ is a coset of $i^{*}[E, \Omega L]_{D}$ in $[F, \Omega T]$. This is the same definition as in [8].

Now we return to the situation at the beginning of the section. Let $f: X \rightarrow Z \in \operatorname{Top}(C \rightarrow D)$ and $h: Z \rightarrow L \in \operatorname{Top}(D=D)$. We have

$$
\left(P_{f}, P_{f}\right) \rightarrow\left(X, P_{f}\right) \rightarrow(Z, D) \rightarrow(L, L)
$$

From [7] we get the following.

$$
\begin{aligned}
{\left[\left(X, P_{f}\right) ;(\hat{\Omega} Z, D)\right]_{Z} } & \longrightarrow\left[\left(X, P_{f}\right) ;(\Omega L, \Omega L)\right] \\
\downarrow & \downarrow \\
{\left[\left(P_{f}, P_{f}\right) ;(\hat{\Omega} Z, D)\right]_{Z} } & \longrightarrow\left[\left(P_{f}, P_{f}\right) ;(\Omega L, \Omega L)\right] \\
{\left[\left(X, P_{f}\right) ;(P, \Omega L)\right]_{D} } & \longrightarrow\left[\left(X, P_{f}\right) ;(Z, D)\right]_{D} \longrightarrow\left[\left(X, P_{f}\right) ;(L, L)\right]_{D} \\
\downarrow & \downarrow \\
& \downarrow \\
\left.\quad\left(P_{f}, P_{f}\right) ;(P, \Omega L)\right]_{D} & \longrightarrow\left[\left(X, P_{f}\right) ;(Z, D)\right]_{D} \longrightarrow\left[\left(P_{f}, P_{f}\right) ;(L, L)\right]_{D}
\end{aligned}
$$

This simplifies to the following.

$$
\begin{aligned}
& {\left[\left(X, P_{f}\right) ;(\hat{\Omega} Z, D)\right]_{Z} \longrightarrow[X, \Omega L]_{D}} \\
& 0 \\
& \longrightarrow\left[P_{f}, \Omega L\right]_{D}
\end{aligned}
$$

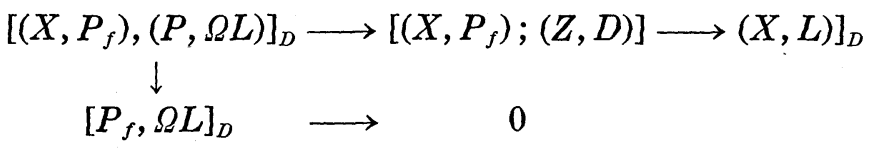

1.3 Definition. Let $s^{\prime}: P_{f} \rightarrow D \rightarrow \Omega_{D} L$ be the composition of the structure maps. Define $\rho:[Z, L]_{D}^{D} \rightarrow\left[P_{f}, \Omega_{D} L\right]_{D}^{C}$ by $\rho(h)=\Gamma\left(s^{\prime} ; f\right)$. 
It follows from Theorem 1.2 that $\rho(h)$ is a coset of $p^{*}\left[X ; \Omega_{D} L\right]$ in $\left[P_{f} ; \Omega_{D} L\right]_{D}$. More concretely, consider

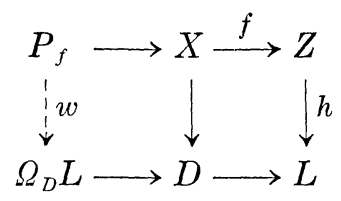

Let $H: X \rightarrow P_{D} L$ be a homotopy of $\check{k} \hat{x}$ to $h f$ in Top $(C \rightarrow D)$. Define $w(x, m)=(P h) m-H(x)$. Then $w \in \rho(h)$ and it is, in fact, a typical element.

Now suppose that $\phi: \Pi \rightarrow$ Aut $G$ is a homomorphism where $\Pi$ is a group and $G$ is an abelian group. Suppose that $D \rightarrow K(\Pi, 1)$ is given, defining a local coefficient system $G_{\phi}$ on $D$ and hence on $P, X$, and $Z$. Use these coefficients and form the following diagram.

$$
\begin{aligned}
\cdots H^{t}(X, C) & \longrightarrow H^{t}(P, C) \\
\uparrow & \longrightarrow H^{t+1}(X, P) \\
\cdots H^{t}(Z, C) \longrightarrow H^{t}(D, C) & \longrightarrow H^{t+1}(Z, D) \\
& \longrightarrow H^{t+1}(X, C) \longrightarrow H^{t}(P, C) \longrightarrow \cdots \\
& \longrightarrow H^{t+1}(Z, C) \longrightarrow H^{t}(D, C) \longrightarrow \cdots
\end{aligned}
$$

Here, and elsewhere in cohomology, a "pair" $(X, A)$ is to be interpreted as the mapping cone of whatever natural map $A \rightarrow X$ is indicated by the context.

1.4 Definition. $R: H^{t+1}\left(Z, D ; G_{\psi}\right) \rightarrow H^{t}\left(P, C ; G_{\phi}\right)$ is defined by $R=$ $S^{-1} \bar{f}^{*}$.

$R$ is a secondary cohomology operation and its indeterminacy is $p^{*} H^{t-1}(X, C) \subset H^{t-1}(P, C)$. Now take $L^{\prime}=L_{\psi}(G, t+1)$, the classifying space for local coefficient cohomology, and $L=D \times_{K} L^{\prime}$. What follows is also valid if $L^{\prime}$ is replaced by a product over $K$ of such spaces. We have

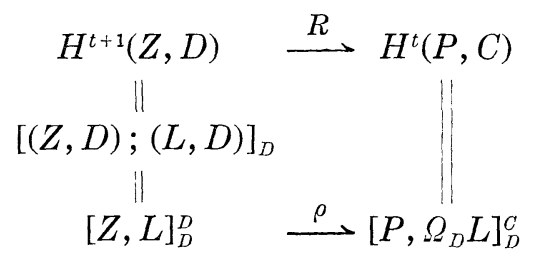


1.5 THEOREM. $R=\rho$.

Proof. Let $h: Z \rightarrow L$. The indeterminacies of $R(h)$ and $\rho(h)$ are the same so it suffices to find a common element. Consider the following diagram.

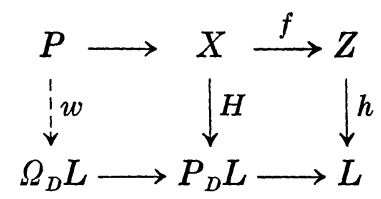

$H$ is a null homotopy of $h f$ (i.e. a homotopy of $\check{k} \hat{x}$ to $h f$ ) and $w$ is the naturally induced map. It is convenient to think of the bottom row as a principal fibration. First of all, it is clear that $R$ is natural for such maps of relative principal fibrations. Secondly, note that if $\lambda(t+1) \epsilon$ $H^{t+1}\left(L, D ; G_{\phi}\right)$ is a fundamental class for $L$ then $\lambda(t) \in R(\lambda(t+1))$. Hence $R(h)=R\left(h^{*} \lambda(t+1)\right) \supset w^{*} R(\lambda(t)) \ni w$. However, it is immediate from the defining diagram for $\rho$ that $w \in \rho(h)$.

Q.E.D.

This proof should be compared to the proof that $\Sigma=\sigma$ in [8]. With some slight awkwardness it would be possible to define a homotopy operation including both $\sum$ and $\rho$ as special cases and prove a theorem which would specialize to both Theorem 1.5 and Theorem 3.1 of [8]. Both operations can be viewed as versions of the bracket operation of Section 5 of [6].

We are interested in finding sufficient conditions for $\rho(=R)$ to be onto. Now assume $\hat{z}: Z \rightarrow D$ is a fibration in Top (pt) and that its fiber is $(n-1)$-connected and that the map $\hat{x}: X \rightarrow D$ is $b$-connected.

1.6 THEOREM. $t \leq \min (2 n-3, n+b-1)$ implies $R$ onto,

$$
R: H^{t}\left(Z, D ; G_{\phi}\right) \longrightarrow H^{t-1}\left(P, C ; G_{\phi}\right) \text {. }
$$

Proof. In the defining diagram of $R$ above it suffices to show $\bar{f}^{*}$ is isomorphic. Since $Z \rightarrow D$ is a fibration in Top (pt) so are $P_{D} Z \rightarrow Z$ and $P \rightarrow X$. We have

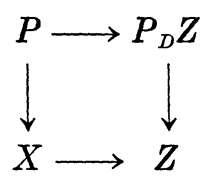

The fibers in Top (pt) of $P \rightarrow X$ and $P_{D} Z \rightarrow Z$ are the same. It follows 
from the $3 \times 3$ lemma (Nomura [10]) that the "fibers" of $P \rightarrow P_{D} Z$ and $X \rightarrow Z$ are homotopically equivalent and so the relative Serre theorem [8] can be applied to $(X, P) \rightarrow\left(Z, P_{D} Z\right)$ and hence to $(X, P) \rightarrow(Z, D)$. It is easy to see that the Top (pt) "fiber" of $X \rightarrow Z$ is $\min (n-2, b)$-connected and $H^{i}(Z, D ;-)=0$ for $i<n$. The relative Serre theorem implies that $\bar{f}^{*}$ is isomorphic for $t+1 \leq \min (2 n-2, b+n)$. Q.E.D.

Now suppose there is a commutative diagram

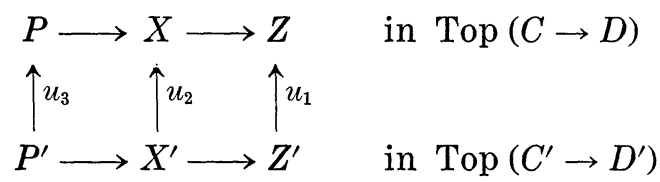

This gives (coefficients $G_{\phi}$ )

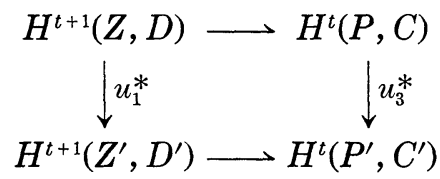

So, in general, $u_{3}^{*} R(g) \subset R^{\prime} u_{1}^{*}(g)$.

1.7 THEOREM. Let $w \in H^{t}(P, C)$. Assume $u_{3}^{*} w \in R^{\prime}\left(u_{1}^{*} g\right)$ for some $g$. Assume $u^{*}: H^{t+1}(X, P) \rightarrow H^{t+1}\left(X^{\prime}, P^{\prime}\right)$ is onto. Then $w \in R(g)$.

Proof. $u^{*} f^{*} g=f^{*} u_{1}^{*} g=\delta^{\prime} u_{3}^{*} w=u^{*} \delta w$. Hence $f^{*} g=\delta w$ and $w \in R(g)$.

Consider now an ordinary principal fibration, in Top $(C \rightarrow \mathrm{pt}), P(u)$ $\rightarrow X \rightarrow Z$ where $u: X \rightarrow Z$. If a map $\hat{x}: X \rightarrow D$ is given then a twisted suspension operation $\rho: H^{n}(D \times Z, D ; G) \rightarrow H^{n-1}(P, C ; G)$ can be defined as follows. First consider $u^{\prime}=u(\hat{x}, u): X \rightarrow D \times Z$ and form the $D$ relative principal fibration $P\left(u^{\prime}\right) \rightarrow X \rightarrow D \times Z$. Then $P\left(u^{\prime}\right)=P(u)$. (Here it might be better to write $P_{D}\left(u^{\prime}\right)$ instead of $P\left(u^{\prime}\right)$.) Now suppose $N \in$ Top (pt) and set $L=D \times N$.

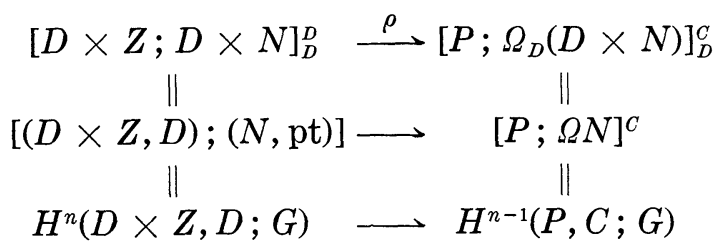

The last two rows give the twisted suspension operation in terms of the original data. The last row assumes $N=K(G, n)$. This transference 
technique can be generalized as follows. Given a $K$-relative principal fibration, a map $\hat{x}: X \rightarrow D$, and $N \in$ Top $(K=K)$, we get a twisted operation

$$
\begin{gathered}
{\left[\left(D \times_{K} Z, D\right) ;(N, \mathrm{pt})\right]_{K}^{p t} \longrightarrow} \\
\| \\
\left.H^{n}\left(D \times_{K} Z, D ; G_{\phi}\right) \longrightarrow \Omega_{K} N\right]_{N}^{C} \\
\|
\end{gathered}
$$

The last row assumes $N=L_{\phi}(G, n)$ and $K=K(\Pi, 1)$.

\section{Reducing Two Story Towers.}

Suppose that the following tower in Top $(C \rightarrow D)$ is given.

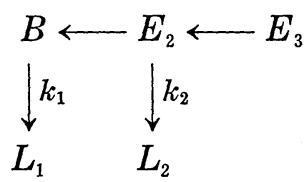

Here $L_{i} \in \operatorname{Top}(D=D)$ and $E_{i}$ is the $D$-relative principal fibration induced by $k_{i}$. We are interested in finding $M \in \operatorname{Top}(D=D)$ and $f: B \rightarrow$ $M$ so that $E_{3} \rightarrow B$ is homotopically equivalent in $\operatorname{Top}(C \rightarrow B)$ to $P_{f} \rightarrow B$ (the $D$-relatively principal fibration induced by $f$ ). The particular example to keep in mind is the following one: $L_{1}^{\prime}=L_{\phi}(G, n), L_{2}^{\prime}=L_{\psi}(H, t)$, $L_{i}=D \times_{K} L_{i}^{\prime}, D \rightarrow K=K(\Pi, 1)$ is a fixed map, $\Pi$ is a group, $G$ and $H$ are abelian groups and $\phi: \Pi \rightarrow \operatorname{Aut}(G)$ and $\psi: \Pi \rightarrow$ Aut $(H)$ are homomorphisms. The main homotopy theoretic result of this section is the following one.

2.1 ThEOREM. Assume $L_{2}=\Omega_{D} J$ and $k_{2} \in \operatorname{Im} \rho:\left[L_{1} ; J\right]_{D}^{D} \rightarrow\left[E_{2} ; L_{2}\right]_{D}^{C}$. Then there is an $M \in \operatorname{Top}(D=D)$ and $f: B \rightarrow M \in \operatorname{Top}(C \rightarrow D)$ such that $P_{f} \rightarrow B$ and $E_{3} \rightarrow B$ are homotopically equivalent in Top $(C \rightarrow B)$.

The theorem will be deduced from a couple of lemmas. First consider the following diagram in Top $(C \rightarrow D)$.

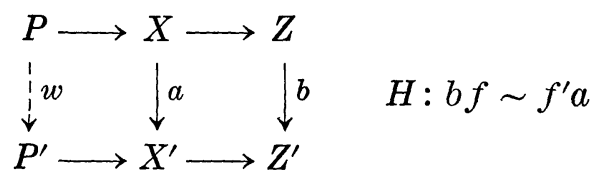

Here $P$ and $P^{\prime}$ are the induced $D$-relative principal fibrations. $H: X \rightarrow$ $W_{D} Z^{\prime}$ is a given homotopy and $w=w_{H}: P \rightarrow P^{\prime}$ is defined by $w(x, m)=$ $(a(x),(P b) m+H(x))$. If $C=D=$ pt then the properties of $w$ are known 
[Nomura, 9]. These known results can be generalized to the present setting without difficulty. In particular, the following lemma can be proved.

2.2 LEMMA. If $a$ and $b$ are homotopy equivalences then so is $w$. If, in addition, $a=\mathrm{id}: X \rightarrow X$ then $w$ is a homotopy equivalence in Top $(C \rightarrow X)$, i.e., a fiber homotopy equivalence in Top $(C \rightarrow D)$.

Now consider the following commutative diagram in Top $(C \rightarrow D)$.

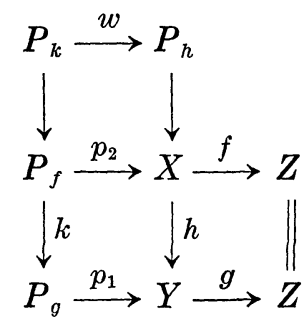

Assume that $g: Y \rightarrow Z \in \operatorname{Top}(D=D)$ so that $P_{g} \in \operatorname{Top}(D=D), g h=f$, $k=(h, \mathrm{id})$, so $h p_{2}=p_{1} k$ and $w=\left(p_{2}, P p_{1}\right)$ here.

2.3 LEMma. The map $w$ is a homotopy equivalence. In fact $w$ is a fiber homotopy equivalence of $P_{k} \rightarrow X$ to $P_{h} \rightarrow X$.

Proof. If $C=D=\mathrm{pt}$ this is a result of Nomura [9]. His proof carries over to the present setting without difficulty. The lemma can also be deduced from a general $3 \times 3$ lemma.

We will now combine the previous two lemmas to get a proof of the main theorem. Consider the following diagram.

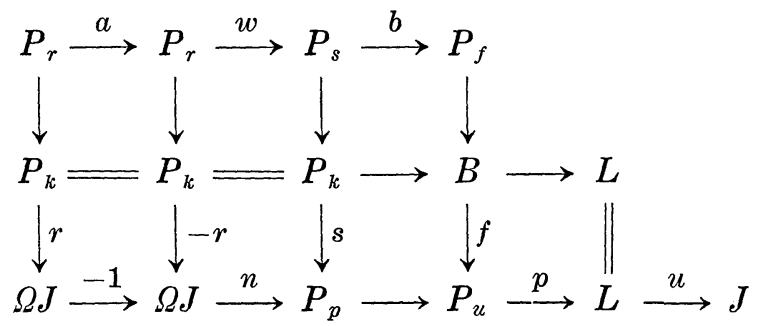

Here $L=L_{1}, k=k_{1}, r=k_{2} \in \rho(u) . \quad r$ is defined by means of some nullhomotopy of $u k$. Use the same null-homotopy to define $f . \quad n$ is the natural homotopy equivalence and $s=(f$, id $)$ and $n(-r) \sim s$ by $H$, say, as is readily checked; all other squares are strictly commutative; the 
map $w$ is due to the homotopy $H . \quad b$ is a homotopy equivalence in Top $(C \rightarrow B)$ by Lemma 2.3 and $a$ and $w$ are homotopy equivalences in Top $\left(C \rightarrow P_{k}\right)$ by Lemma 2.2. Hence bwa is a homotopy equivalence in Top $(C \rightarrow B)$. Take $M=P_{u}$. This completes the proof of Theorem 2.1.

If $C=D=\mathrm{pt}$, then 2.1 is related to Lemma 1.6 of Gershenson [2]. In order to apply 2.1 we need some conditions which guarantee that $k_{2}$ is in the image of $\rho$. For simplicity we consider only the specific situation described at the beginning of the section.

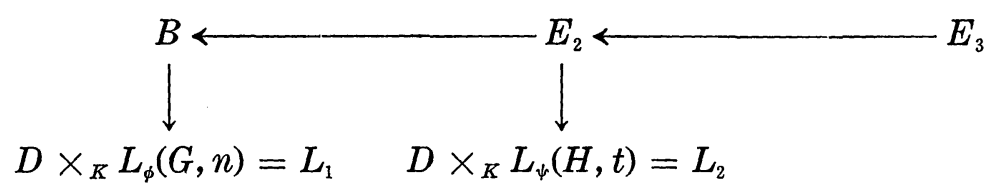

Here $n \leq t$. The results below can, however, be stated in more general terms and proved by the same methods. In particular $L(G, n)$ can be replaced by $\Pi_{K} L\left(G_{i}, n_{i}\right)$ and $L(H, t)$ by $\Pi_{K} L\left(H_{j}, t_{j}\right)$. It is now assumed that all spaces involved have the homotopy type of $C W$ complexes.

2.4 Corollary. Assume $B \rightarrow D$ is b-connected and that $t \leq$ $\min (2 n-3, n+b-1)$. Then there is an $M \in \operatorname{Top}(D=D)$ and $f: B \rightarrow$ $M \in \operatorname{Top}(C \rightarrow D)$ such that $P_{f} \rightarrow B$ and $E_{3} \rightarrow B$ are homotopically equivalent in Top $(C \rightarrow B)$.

Proof. Theorem 2.1, 1.5, and 1.6 give this. Here

$$
J=D \times_{K} L_{\psi}(H, t+1) .
$$

Now the above diagram is enlargened as follows.

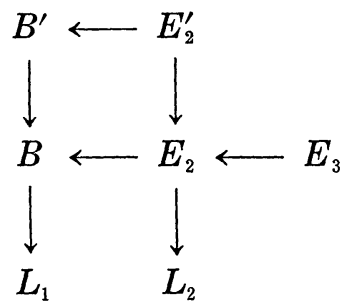

$E_{2}^{\prime}$ is the pullback of $E_{2} \rightarrow B$. We have

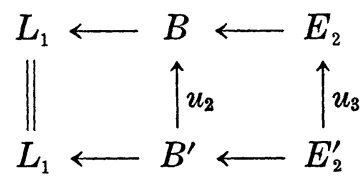


and hence

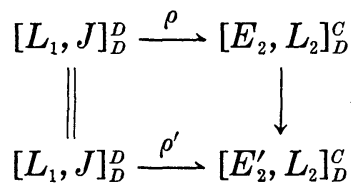

Let $k_{2}^{\prime}$ be the composition $E_{2}^{\prime} \rightarrow E_{2} \rightarrow L_{2}$.

2.5 THEOREM. Assume $k_{2}^{\prime} \in \operatorname{Im} \rho^{\prime}$ and that $t \leq n+b$ where $b=$ connectivity of $B^{\prime} \rightarrow B$. Then the conclusion of 2.4 is valid.

Proof. It suffices, by 2.1 , to show $k_{2} \in \operatorname{Im} \rho$. Theorem 1.7 will be used for this purpose. Recall, Theorem 1.5, $\rho=R$ and $\rho^{\prime}=R^{\prime}$, so:

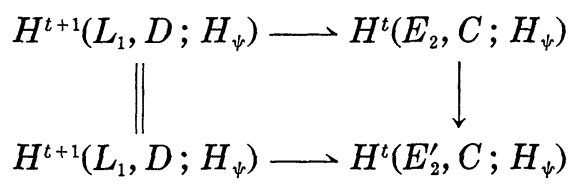

and $k_{2} \in H^{t}\left(E_{2}, C\right), \quad u_{3}^{*} k_{2}=k_{2}^{\prime} \in \operatorname{Im} R^{\prime}$. We must only establish that $u^{*}: H^{t+1}\left(B, E_{2}\right) \rightarrow H^{t+1}\left(B^{\prime}, E_{2}^{\prime}\right)$ is a monomorphism. Since $L_{1} \rightarrow D$ is a fibration in Top (pt), so are $E_{2} \rightarrow B$ and $E_{2}^{\prime} \rightarrow B^{\prime}$ and these last two have the same fiber. It follows from the $3 \times 3$ lemma in Top (pt) that $B^{\prime} \rightarrow$ $B$ and $E_{2}^{\prime} \rightarrow E_{2}$ have homotopically equivalent "fibers" and hence that the relative Serre theorem [8] can be applied to $\left(B^{\prime}, E_{2}^{\prime}\right) \rightarrow\left(B, E_{2}\right)$. If it can be shown that $H^{p}\left(B, E_{2} ; H^{q}(F ; H)_{\psi}^{\sim}\right)=0$ for $p<m$ or $0<q<m^{\prime}$ and $t$ $+1 \leq m+m^{\prime}$ it will then follow that $u^{*}$ is monomorphic. By assumption $F$ is $b$-connected so $m^{\prime}=b+1$. Now consider $H^{p}\left(L_{1}, D ; \Gamma\right) \rightarrow$ $H^{p}\left(B, E_{2} ; \Gamma\right)$ where $\Gamma$ is any local coefficient system. Just as above we see that the "fibers" of $B \rightarrow L_{1}$ and $E_{2} \rightarrow D$ are homotopically equivalent, so the relative Serre spectral sequence can be applied. But $H^{p}\left(L_{1}, D ; \Gamma\right)$ $=0$ for $p<n$ so it follows that the same is true of $H^{p}\left(B, E_{2} ; \Gamma\right)$. Hence $m=n$ and $u^{*}: H^{i}\left(B, E_{2} ; H_{\psi}\right) \rightarrow H^{i}\left(B^{\prime}, E_{2}^{\prime} ; H_{\psi}\right)$ is isomorphic for $i \leq n+$ $b-1$ and monomorphic for $i \leq n+b+1$ Q.E.D.

Some special cases are of interest. First note that if $B=B^{\prime}$ then 2.5 reduces to 2.1 . Next, take $B^{\prime}=E_{3}$.

2.6 CoRollary. Suppose $B^{\prime}=E_{3}, k_{2}^{\prime} \in \operatorname{Im} \rho^{\prime}$. Then if $t \leq 2 n-2$ the conclusion of 2.4 is valid. 
Proof. Since $L_{i} \rightarrow D$ is a fibration in Top (pt), $i=1,2$, it follows that $E_{3} \rightarrow B$ is also and is an extension of $K(G, n-1)$ by $K(H, t-1)$. Since $n \leq t$, we see that $E_{3} \rightarrow B$ is $(n-2)$-connected and the corollary follows from Theorem 2.5.

Next, take $C=B^{\prime}=D$. Then there is the following diagram in $\operatorname{Top}(D=D)$.

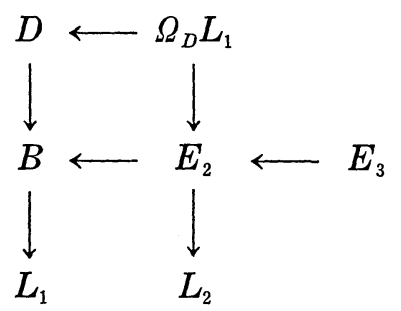

Here $\Omega_{D} L_{1}=\Omega_{D}\left(D \times{ }_{K} L(G, n)\right)=D \times_{K} L(G, n-1)$. Let $k_{2}^{\prime}$ be the composite $\Omega_{D} L_{1} \rightarrow E_{2} \rightarrow L_{2}$.

2.7 CoRollary. Suppose $k_{2}^{\prime} \in \operatorname{Im} \Omega_{D}:\left[L_{1}, J\right] \rightarrow\left[\Omega_{D} L_{1}, L_{2}\right]$. Suppose also that $B \rightarrow D$ is r-connected and $t \leq n+r-1$. Then there is an $f: B \rightarrow$ $M \in \operatorname{Top}(D=D)$ such that $P_{f} \rightarrow B$ and $E_{3} \rightarrow B$ are homotopically equivalent in $\operatorname{Top}(D \rightarrow B)$.

Proof. Consider

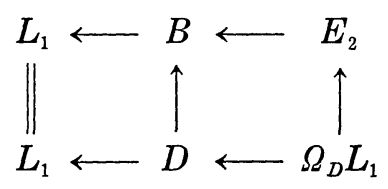

It is clear from the definition of $\rho$ that $\rho=\Omega_{D}$. Also, since $B \rightarrow D$ is a retraction and is $r$-connected it follows that $D \rightarrow B$ is $(r-1)$-connected. Hence 2.7 follows from 2.5.

Consider now a tower of ordinary principal fibrations is Top $(C \rightarrow \mathrm{pt})$.

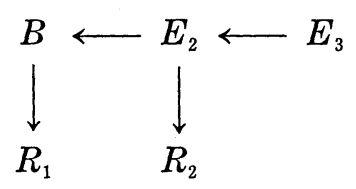

Assume $\hat{b}: B \rightarrow D$ is given and $R_{2}=\Omega R_{2}^{\prime}$. Then a sufficient condition for $E_{3} \rightarrow B$ to be $D$-relatively principal is that $r_{2} \in \operatorname{Im} \rho:\left[\left(D \times R_{1}, D\right) ;\left(R_{2}^{\prime}, \mathrm{pt}\right)\right]$ 
$\rightarrow\left[E_{2}, R_{2}\right] . \quad \rho$ is the operation discussed at the end of Section 1 . This follows from 2.1 because we can consider

$$
\begin{aligned}
& B \longleftarrow E_{2} \longleftarrow E_{3} \\
& \downarrow\left(\hat{b}, r_{1}\right) \quad \downarrow\left(\hat{b}, r_{2}\right) \\
& D \times R_{1} \quad D \times R_{2}
\end{aligned}
$$

and it is a tower of $D$-principal fibrations so 2.1 applies. More generally, one can transfer from Top $(C \rightarrow K)$ to Top $(C \rightarrow D)$ and this is what was done implicitly in 2.4 .

\section{Reducing Towers}

I want to give a version of 2.1 for higher towers. Consider the following tower of $D$-relatively principal fibrations.

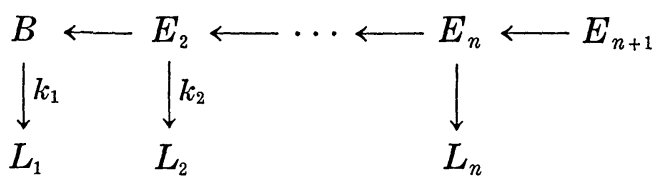

In this section write $P(f)$ for $P_{f}$. For the operation $\rho$ of 1.3 write $\rho(f: h)$ instead of $\rho(h)$. In the proof of Theorem 2.1 denote $f$ by $k_{2}^{\prime}$. Thus we have the diagram

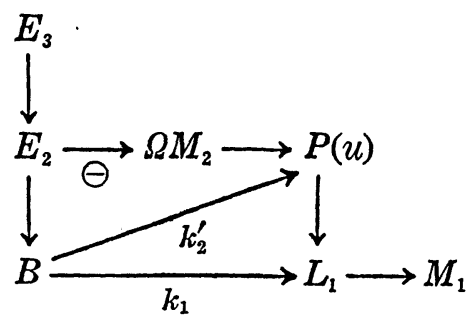

where $\Omega$ means $\Omega_{D}$ and $\Omega M_{2}=L_{2}$. The conclusion of 2.1 is that $E_{3} \rightarrow$ $B$ and $P\left(k_{2}^{\prime}\right)$ are homotopy equivalent in Top $(C \rightarrow B)$. Identify $E_{3}$ and $P\left(k_{2}^{\prime}\right)$ by the equivalence of the proof of Theorem 2.1. Consider the following statements $\left(A_{i}\right)$ for $i \geq 2$.

$$
\begin{array}{ll}
k_{i} \in \rho\left(k_{i-1}^{\prime} ; v_{i-1}\right) & \text { where } v_{i-1}: P_{i-1} \rightarrow M_{i-2}, \\
P_{i-1}=P\left(v_{i-2}\right), & \Omega M_{i-2}=L_{i-1} .
\end{array}
$$

If $i=2$ interpret $P_{i}$ as $L_{1}$. 
3.1 THEOREM. Assume $\left(A_{i}\right)$ for $2 \leq i \leq n$. Then $E_{n+1} \rightarrow B$ is equivalent to $P\left(k_{n}^{\prime}\right) \rightarrow B$ in $\operatorname{Top}(C \rightarrow B)$.

Proof. If $A_{i-1}$ is true then $P_{i-1}=P\left(v_{i-2}\right)$ can be formed and $v_{i-1}: P_{i-1}$ $\rightarrow M_{i-2}$ with $k_{i} \in \rho\left(k_{i-2}^{\prime} ; v_{i-2}\right)$ can be selected. We can form $k_{i-1}^{\prime}$ and identify $E_{i-1} \rightarrow B$ with $P\left(k_{i-1}^{\prime}\right) \rightarrow B$. Hence $A_{i}$ makes sense. $A_{2}$ is true so 3.1 follows from 2.1 by induction.

From now on assume all spaces have homotopy type of $C W$ complexes.

3.2 THEOREM. Assume $L_{i}=D \times{ }_{K} L_{\phi}\left(H_{i}, t_{i}\right)$ where $D \rightarrow K=(\Pi, 1)$ is given. Assume $t_{1} \leq t_{2} \leq \cdots \leq t_{n}$ and $t_{i} \leq \min \left(2 t_{1}-3, t_{1}+b+1\right)$ where $b=$ connectivity of $B \rightarrow D$. Then there is an $M \in \operatorname{Top}(D=D)$ and $f: B$ $\rightarrow M \in \operatorname{Top}(C \rightarrow D)$ with $P(f) \rightarrow B$ and $E_{n+1} \rightarrow B$ homotopically equivalent in $\operatorname{Top}(C \rightarrow B)$.

Proof. Assume $\left(A_{i-1}\right)$ has been established. $H^{j}\left(L_{1}, D ;-\right)=0, j<t_{1}$, plus the Serre spectral sequence gives $H^{j}\left(P_{m}, D ;-\right)=0, j<t_{1}, m \leq i-1$. The proof of Theorem 1.6 now gives $\left(A_{i}\right)$ Q.E.D.

3.3 CoRollary. Assume $L_{i}=D \times_{K} K_{\phi}\left(H_{i}, t_{i}\right), t_{1} \leq t_{2} \leq \cdots \leq t_{n} \leq 2 t_{1}$ -3. Then the conclusion of 3.2 is valid with $D=B$.

Proof. $b=\infty$ in 3.2.

Note that in 3.2 and 3.3 we can take $L_{i}=D \times_{K} \Pi_{K} L_{\phi}\left(H_{i, j}, t_{i, j}\right)$ provided $t_{i}=t_{i, 1} \leq t_{i, 2} \leq \cdots$. This last corollary is related to a result of Larmore [4].

3.4 CoRollary. Let $p: E \rightarrow B$ be a fibration in Top (pt) with fiber $F=p^{-1}\left(b_{0}\right)$. Assume $\Pi_{i}(F)=0$ except possibly when $s \leq i<2 s-1$. Then there is an $M \in \operatorname{Top}(B=B)$ and $f: B \rightarrow M$ such that $P(f) \rightarrow B$ and $E \rightarrow$ $B$ are homotopically equivalent in Top $(C \rightarrow B)$.

Proof. Let the diagram at the beginning of the section come from the Postnikov factorization of $p$ (see Section 4 of [8]). Thus $L_{1}=B \times_{K}$ $L\left(\Pi_{s} F, s+1\right), \cdots, L_{n}=B \times{ }_{K} L\left(\Pi_{2 s-2} F, 2 s-1\right), n=s-1, t_{1}=s+1$, and $t_{i} \leq 2 s-1=2 t_{1}-3$. Hence 3.4 follows from 3.3.

Note that 3.3 is actually valid with $D=B^{\prime}$ where $B \rightarrow B^{\prime}$ is $b$-connected, $b \geq t_{1}-2$. So in 3.4 we can take $M \in \operatorname{Top}\left(B^{\prime}=B^{\prime}\right)$ for such a $B^{\prime}$. For example, if $\cdots \rightarrow B(j) \rightarrow \cdots \rightarrow B(1)=K\left(\Pi_{1} B, 1\right)$ is the Postnikov system for $B$ then $B^{\prime}=B\left(t_{1}-2\right)$ is permissable in 3.3 and $B^{\prime}=B(s-1)$ in 3.4 . 
3.5 Corollary. Let $E \rightarrow B$ be a fibration in Top (pt) with fiber $F$ and $\Pi_{i} F=0$ except possibly when $s \leq i<2 s-1$. Assume $B$ is s-connected. Then $E \rightarrow B$ is fiber homotopically equivalent to a principal fibration.

Proof. $D=$ pt in the above comment.

Corollary 3.5 is known and is, in fact, a special case of a theorem of Ganea [1] and Hilton [3]. In order to generalize the Ganea-Hilton theorem we consider the following diagram in Top $(D=D)$.

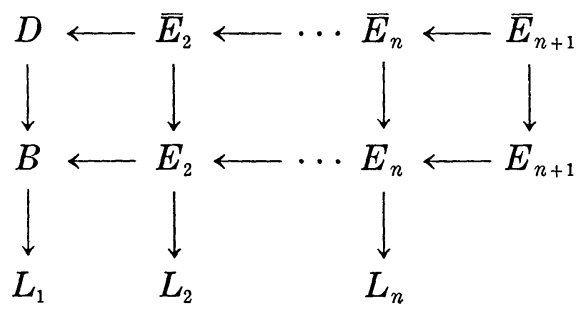

The top row is obtained by pullback from the middle row. Let $\bar{k}_{i}$ be the composite $\bar{E}_{i} \rightarrow E_{i} \rightarrow L_{i}$.

3.6 Theorem. Assume $B \rightarrow D$ is r-connected, $\bar{k}_{i} \in \operatorname{Im} \Omega_{D}:\left[\hat{E}_{i} ; M_{i}\right] \rightarrow$ $\left[\bar{E}_{i}, L_{i}\right]$, where $\Omega \hat{E}_{i}=\bar{E}_{i}$, and $t_{i} \leq t_{1}+r+1,1 \leq i \leq n$. Then there is an $f: B \rightarrow M \in \operatorname{Top}(D=D)$ such that $P(f) \rightarrow B$ and $E_{n+1} \rightarrow B$ are homotopically equivalent in Top $(D \rightarrow B)$. Moreover, $\Omega_{D} M=\bar{E}_{n+1}$.

Proof. This follows from 3.1 just as 3.7 followed from 2.1.

3.7 Theorem. Assume $p: E \rightarrow B \in$ Top $(D=D)$ and is a fibration in Top (pt) with fiber $F=p^{-1}\left(b_{0}\right)$. Let $\bar{E}$ be the pullback of $p$ and $\breve{b}: D \rightarrow$ $B$. Assume $\bar{E}=\Omega_{D} Z$ for some $Z \in \operatorname{Top}(D=D)$ and $B \rightarrow D$ is r-connected. Assume $\Pi_{i} F=0$ except possibly when $s \leq i<s+r$. Then there is an $f: B \rightarrow Z$ in Top $(D=D)$ such that $P(f) \rightarrow B$ and $E \rightarrow B$ are homotopically equivalent in Top $(D \rightarrow B)$.

Proof. Let the above tower come from the Moore-Postnikov factorization of $p$. The $\bar{E}$-tower is then the Postnikov tower for $\bar{E}_{n+1} \rightarrow D$. However, this can also be obtained by applying $\Omega_{D}$ to the Postnikov tower for $Z \rightarrow D$. It follows that each $\bar{k}_{i}$ is indeed in the image of $\Omega_{D}$ (by "uniqueness" of Postnikov invariants). Here $t_{1}=s+1$ and $t_{i} \leq s$ $+r=t_{1}+r-1$. The result now follows from 3.6. $M$ can be taken 
to be $Z$ because at each stage of the inductive construction the $v_{i}$ can be taken to be the Postnikov invariant of $Z$.

The Ganea-Hilton result is the case $D=$ pt of 3.7. Finally we describe a version of 3.1 which does not explicitly require Top $(D=D)$ language. Consider a tower of ordinary principal fibrations (in Top $C$ $\rightarrow \mathrm{pt})$.

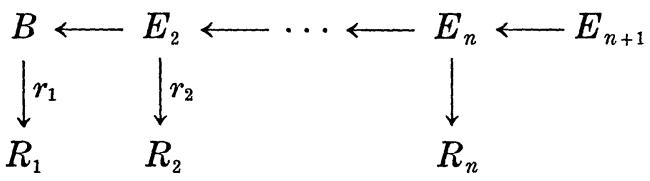

Assume $\hat{b}: B \rightarrow D$ is given. For simplicity take $R_{i}=K\left(G_{i}, t_{i}\right)$.

$\begin{array}{ll}\left(A_{i}\right) \quad r_{i} \in \operatorname{Im} \rho: H^{t_{i}+1}\left(P_{i-1}, D ; G_{i}\right) \rightarrow H^{t_{i}}\left(E_{i}, C ; G_{i}\right) \quad \text { where } P_{i-1}=P\left(v_{i-2}\right), \\ & v_{i-2}:\left(P_{i-2}, D\right) \rightarrow\left(R_{i}^{\prime}, \mathrm{pt}\right) \text { and } \Omega R_{i}^{\prime}=R_{i} .\end{array}$

If follows from 3.1 that $\left(A_{i}\right)$ for $2 \leq i \leq n$ gives $E_{n+1} \rightarrow B$ a $D$-relatively principal fibration (see the end of Section 2). There is a similar local coefficient formulation.

\section{REFERENCES}

[1] T. Genea, Fibrations and cocategory, Comment. Math. Helv. 35 (1961) 15-24.

[2] H. H. Gershenson, Higher composition products, J. Math. Kyoto Univ. 5 (1965), $1-37$.

[ 3 ] P. J. Hilton, On excision and principal fibrations, Comment. Math. Helv. 35 (1961), 77-84.

[4] L. L. Larmore, Twisted cohomology theories and the single obstruction to lifting, to appear.

[5] J. F. McClendon, Thesis, Berkeley (1966) (Abstract published in Dissertation Abstracts, February, 1967, 2785-8).

[6] _ Higher order twisted cohomology operations, Inventiones math., 7 (1969), 183-214.

[7] - Relative principal fibrations, to appear.

[ 8 ] - Obstruction theory in fiber spaces, Math. Zeit. 120 (1971), 1-17.

[ 9 ] Y. Nomura, On mapping sequences, Nagoya Math. J. 17 (1960), 111-145.

[10] —, The Whitney join and its dual, Osaka J. Math. 7 (1970), 353-373. 